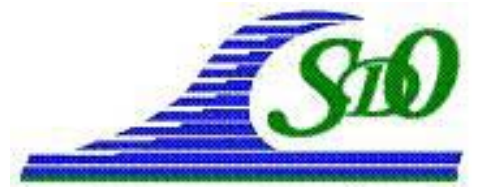

XI ${ }^{\text {èmes }}$ Journées Nationales Génie Côtier - Génie Civil

Les Sables d'Olonne, 22-25 juin 2010

DOI:10.5150/jngcgc.2010.011-F @ Editions Paralia CFL

disponible en ligne - http://www.paralia.fr - available online

\title{
Validation d'un terme source de dissipation par déferlement applicable du large à la côte
}

\author{
Jean-François FILIPOT ${ }^{1}$, Fabrice ARDHUIN ${ }^{1}$, Rudy MAGNE ${ }^{2}$
}

1. Ifremer, Laboratoire d'Océanographie Spatiale, Centre de Brest, 29200 Plouzané, France.

Jean-François.Filipot@ifremer.fr ; Fabrice.Ardhuin@ifremer.fr

2. SHOM, division HOM, 13 rue du Chatellier, 29228 Brest Cedex 2, France.

rmagne@shom.fr

\section{Résumé :}

Un nouveau terme source destiné aux modèles spectraux est présenté. Il a l'avantage de reposer sur des grandeurs physiques pouvant être validées par des observations, notamment sur le paramétrage de la probabilité de déferlement par échelles de vagues développé par FILIPOT et al. (sous presse). La dissipation d'abord calculée dans le domaine physique est ensuite redistribuée sur le spectre. Ce terme source a en outre la particularité de représenter de façon unique la dissipation d'énergie associée au déferlement, du large à la côte. Une estimation de l'efficacité de ce terme source a été réalisée dans diverses situations, de l'échelle globale jusqu'au déferlement bathymétrique et les premiers résultats obtenus sont encourageants.

Mots-clés :

Déferlement - Dissipation - Modèles spectraux - Terme source

\begin{abstract}
:
A new source term intended to spectral models is presented. It has the advantage to be based on physical variables that may be validated by observations, it relies in particular on the breaking probability parameterization per wave scales developped by FILIPOT $e t$ al. (in press). The dissipation, first calculated in the physical space is then distributed over the spectrum. Moreover, this source term represents, in the mean time, the wave breaking energy dissipation from deep ocean to shallow water. The ability of this source term has been tested in various situations from global scale to depth-induced breaking and the first results are encouraging.
\end{abstract}

\section{Keywords:}

Wave breaking - Dissipation - Spectral models - Source term

\section{Introduction}

Bien qu'étant un paramètre clé dans de nombreux domaines (modélisation des états de mer, échanges gazeux à l'interface air mer, etc...), le déferlement et la dissipation d'énergie associée sont encore assez mal compris et très mal quantifiés. En 
conséquence, la modélisation de l'évolution des vagues est largement empirique. D'autre part, une distinction arbitraire est faite entre le paramétrage du déferlement en eau profonde (moutonnement) et du déferlement à la plage. Nous présentons ici un terme source de dissipation basé sur un paramétrage de la probabilité de déferlement par échelles de vagues développé par FILIPOT et al. (sous presse). Pour produire un taux de dissipation spectral, cette probabilité de déferlement doit être associée à une densité de longueur de crête par unité de surface ainsi qu'à un taux de dissipation par unité de longueur de crête. Le paramétrage de ces deux grandeurs sera discuté dans cet article. Après avoir détaillé la construction du nouveau terme source de dissipation, les premiers résultats seront présentés et discutés.

\section{Description de l'approche}

\subsection{Principe général}

Comme annoncé précédemment, ce terme source repose principalement sur les travaux de FILIPOT et al. (sous presse). Ces auteurs font l'hypothèse, qu'au sein d'un champ de vagues coexistent plusieurs échelles caractérisées par différentes probabilités de déferlement. En filtrant le spectre avec une fenêtre appropriée, ils proposent d'obtenir les différentes échelles, auxquelles correspondra une densité de probabilité de hauteurs de vagues déferlantes. Ici, en sus, nous associerons à chaque échelle une longueur de crête par unité de surface et un taux de dissipation par unité de longueur de crête, nous serons alors capables d'estimer le taux de dissipation par unité de surface. Cette dissipation par échelles, calculée dans l'espace physique, sera enfin redistribuée sur les composantes spectrales contribuant aux différentes échelles.

\subsection{Construction du nouveau terme source}

Définissons tout d'abord les échelles de vagues $L_{i}$ :

$$
L_{i}=\frac{2 \pi \int_{0}^{\infty} W_{i}(k) E(k) d k}{2 \pi \int_{0}^{\infty} W_{i}(k) k E(k) d k}
$$

Avec $k$ le nombre d'onde et $E(k)$ le spectre intégré sur les directions. La fenêtre $W_{i}$ est une fenêtre rectangulaire, égale à 1 pour $0.49 \times\left(2 \pi / L_{i}\right)<k<1.69 \times\left(2 \pi / L_{i}\right)$. Cette fenêtre avait été utilisée par ailleurs par BANNER et al. (2000) pour sélectionner les vagues dominantes en eau profonde. Le taux de dissipation par unité de surface dans le domaine physique pour l'échelle $L_{i}, D\left(L_{i}\right)$ s'écrit comme :

$$
D\left(L_{i}\right)=\int_{0}^{\infty} \varepsilon\left(H, L_{i}\right) \times \Lambda\left(L_{i}\right) \times P_{F A B}\left(H, L_{i}\right) d H
$$

Où $P_{F A B}$ est la densité de probabilité de hauteurs de vagues déferlantes définie par FILIPOT et al. (sous presse), $\varepsilon$ le taux de dissipation par unité de longueur de crête et $\Lambda$ la densité de longueur de crête par unité de surface. $D\left(L_{i}\right)$ apparaît donc clairement 
comme un taux dissipation par unité de surface $(\varepsilon \times \Lambda)$, moyenné sur la distribution des hauteurs des déferlantes d'échelle $L_{i}\left(P_{F A B}\right)$.

Le taux de dissipation dans le domaine spectral est finalement obtenu en associant cette quantité $D\left(L_{i}\right)$, aux composantes spectrales qui contribuent à cette échelle $L_{i}$ :

$$
S_{d s 1}(k)=\frac{D\left(L_{i}\right) E(k)}{\int_{0}^{\infty} W_{i}(k) E(k) d k}
$$

Cependant, comme une composante peut contribuer à plusieurs échelles, la fenêtre $W_{i}$ est translatée de manière continue le long de l'axe des nombres d'onde et le taux de dissipation spectral pour la composante $k$ s'exprime comme la moyenne suivante :

$$
S_{d s}(k)=\frac{1}{N(k)} \sum_{i=1}^{N(k)} \frac{D\left(L_{i}\right) E(k)}{\int_{0}^{\infty} W_{i}(k) E(k) d k}
$$

Ici $N(k)$ est le nombre d'échelles auxquelles participe la composante $k$. Nous allons maintenant détailler les paramétrages utilisés pour la densité de hauteurs de déferlantes, le taux de dissipation par unité de longueur de crête et la densité de longueur de crête.

\subsection{La densité de hauteurs de vagues déferlantes}

Nous rappelons ici brièvement la formulation présentée dans FILIPOT et al. (sous presse) :

$$
P_{F A B}\left(H, L_{i}\right)=\left[\frac{\beta_{r}}{\bar{\beta}}\right]^{2}\left\{1-\exp \left[-\left(\frac{\beta}{\bar{\beta}}\right)^{4}\right]\right\} P_{R}\left(H, L_{i}\right)
$$

Où $P_{R}$ est la distribution de Rayleigh, $\beta$ et $\beta_{r}$ sont des paramètres inspirés des travaux de MICHE (1944) et $\bar{\beta}$ une limite pour le déferlement tenant compte de la linéarisation des vagues inhérente au filtrage spectral. La probabilité de déferlement est obtenue par:

$$
Q_{F A B}\left(L_{i}\right)=\int_{0}^{\infty} P_{F A B}\left(H, L_{i}\right) d H
$$

Pour plus de détails sur ce paramétrage le lecteur pourra se référer à FILIPOT et al. (sous presse).

\subsection{Le taux de dissipation par unité de longueur de crête}

Plusieurs théories ont été avancées pour estimer le taux de dissipation dû au déferlement. L'approche dite du "roller" considère le mouton comme une masse inerte dont le poids serait exactement compensé par les tensions turbulentes générées par les vitesses orbitales à l'interface (DUNCAN, 1981). En eau peu profonde, on fait souvent l'analogie avec la perte d'énergie associée au ressaut hydraulique. En supposant que le débit par unité de longueur de crête à travers la déferlante peut être estimé par $C \times D$, (HWANG \& DIVODKY, 1970) où $D$ est la profondeur et $C$ la vitesse de phase, THORNTON \& GUZA (1983) ont proposé un taux de dissipation par unité de longueur de crête qui fut étendu à l'eau profonde par CHAWLA \& KIRBY (2002) : 


$$
\varepsilon=\frac{1}{4}(B H)^{3} \sqrt{\frac{g k}{\tanh (k D)}}
$$

Avec $B$ le rapport de la hauteur du mouton sur la hauteur de la vague. Cette approche est la seule compatible avec notre paramétrage car valide de l'eau profonde à l'eau peu profonde. Toutefois, cette formulation a été modifiée pour tenir compte des variations de la sévérité du déferlement du large à la côte. Le coefficient $B$ est alors remplacé par $B / \tanh (k D)^{n}$, nous faisons ici l'hypothèse que le déferlement bathymétrique est plus dissipatif que le moutonnement au large. La validation sur les données d'eau peu profonde (présentée plus loin) a permis de calibrer $n$ à 1.5. En pratique, le facteur d'efficacité du déferlement sera utilisé comme paramètre d'ajustement du modèle, il est donc important d'avoir une idée des valeurs extrêmes qu'il peut prendre. Après comparaisons de travaux de MELVILLE (1994), DUNCAN (1981), PHILLIPS et al., (2001) et BANNER \& PEIRSON (2007), il apparaît que $B$ pourrait varier de 0.13 à 0.84 .

\subsection{La longueur de crête par unité de surface}

Nous avons fait précédemment l'hypothèse que les vagues d'échelle $L$ étaient formées des composantes spectrales comprises entre $0.49 k$ et $1.69 k$. Considérons maintenant le cas de vagues régulières de longueur d'onde $L$, la densité de longueur de crête par unité de surface et de nombre d'onde associée est alors:

$$
\Lambda^{*}(L)=\frac{k}{2 \pi \Delta k} \approx 0.13
$$

Ce résultat trivial peut par ailleurs être rapproché des observations faites par SCOTT et al. (2005) qui ont mesuré, à la surface de l'océan Pacifique, des valeurs de $\Lambda^{*}$ entre 0.1 à 0.2 ce qui est au final en assez bon accord avec notre précédente estimation. Par ailleurs, nous n'avons besoin ici que de la densité de longueur de crête par unité de surface, c'est à dire: $\Lambda(L)=\Lambda^{*}(L) \Delta k=k / 2 \pi$.

\section{Premières calibrations}

Le terme source a été implémenté dans le modèle spectral WAVEWATCH III ${ }^{\mathrm{TM}}$ dans sa version 3.14 avec un paramétrage de la génération des vagues et de la dissipation modifié par ARDHUIN et al. (2009) (version "TEST441"), seul le terme de déferlement est modifié. Le coefficient $B$ du modèle a été calibré sur un cas académique simple. Le modèle a tout d'abord été forcé par un spectre de Pierson-Moskowitz de fréquence pic $0.12 \mathrm{~Hz}$, correspondant à un état de mer pleinement développé par un vent de $10 \mathrm{~m} / \mathrm{s}$. Un équilibre satisfaisant entre les différents termes source a été atteint pour une valeur de $B$ fixée à 0.185 . Le modèle a ensuite été comparé à l'ensemble des mesures de hauteurs significatives faites par altimètre en 2007 afin d'évaluer le comportement du nouveau paramétrage à l'échelle globale. Ces résultats encourageants (figure 1) 
montrent notamment une forte réduction de l'erreur le long des côtes est (ex : Nouvelle Angleterre, Plateau Argentin) par rapport au paramétrage présenté par ARDHUIN et al. (2009) et ARDHUIN et al. (sous presse). Des zones d'erreurs plus fortes sur les côtes ouest peuvent être liées à une surestimation de l'énergie de la houle comme le montre par ailleurs la carte du biais (figure 1). Cette erreur devrait être corrigée en augmentant l'atténuation de la houle causée par le frottement air-mer, qui est justement sous estimée par ARDHUIN et al. (sous presse).

Grâce au paramétrage de la probabilité de déferlement et du taux de dissipation valide du large à la côte, on peut espérer que le nouveau terme source soit aussi opérationnel en zone côtière. Cet aspect a pu être validé grâce à un jeu de données, collecté lors de la campagne Duck94 au "U.S. Army Corps of Engineers Field Reasearch Facility" (FRF), une description de l'expérience est disponible par exemple dans ELGAR et al. (1997). Les spectres observés en différents points du profil de plage (figure 2) fournissent une estimation de la hauteur rms des vagues à une heure d'intervalle pendant $270 \mathrm{~h}$.

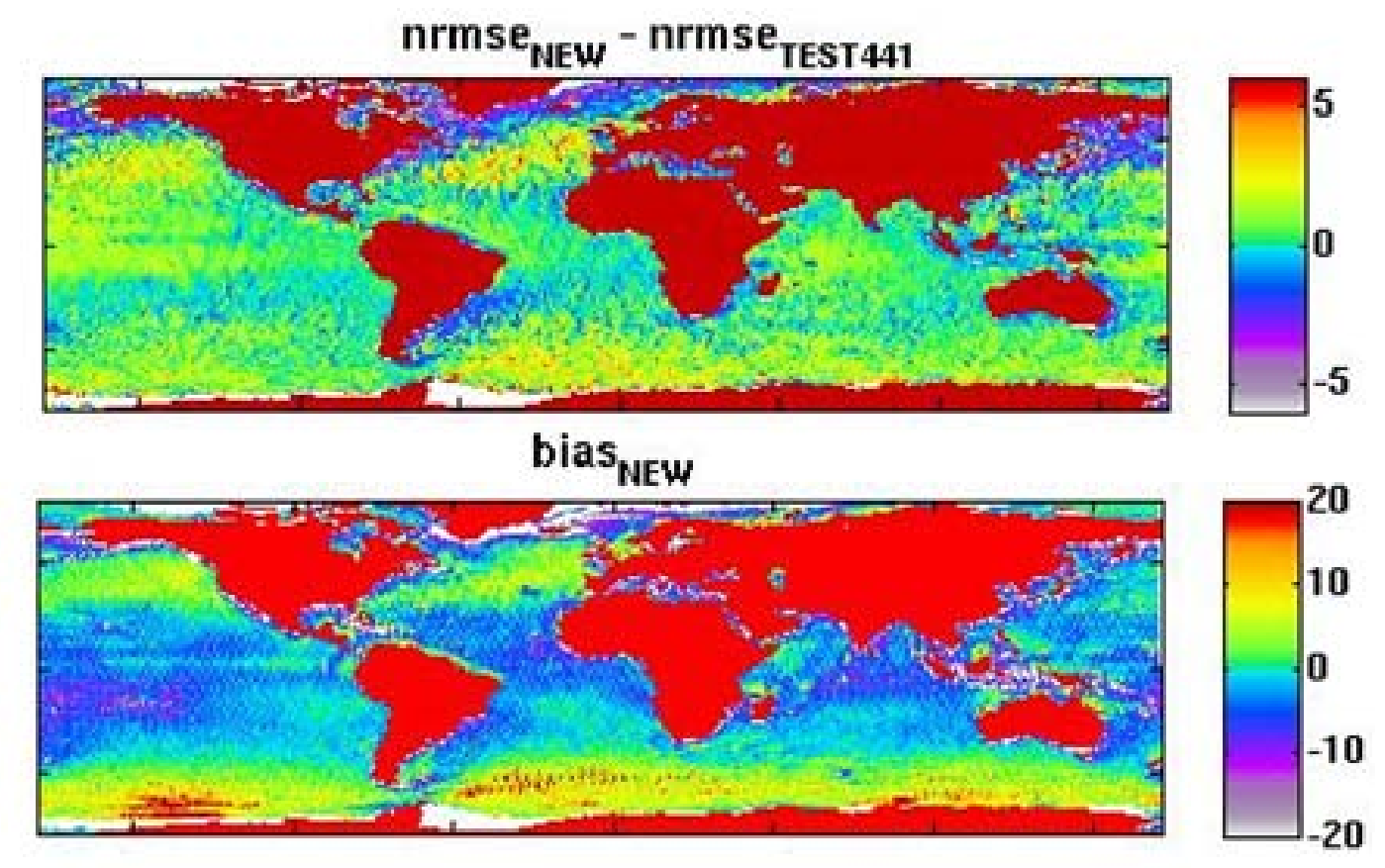

Figure 1. Validation grâce aux mesures altimétriques pour la hauteur significative, pour l'année 2007: en haut comparaison de l'erreur moyenne quadratique normalisée nrms (en \%) du nouveau paramétrage avec celle obtenue avec le TEST441 de ARDHUIN et al. (2009). En bas biais (en \%) du nouveau paramétrage.

La comparaison présentée figure 3 montre un très bon accord entre les hauteurs observées et celles prédites par le modèle. Par ailleurs, deux simulations différentes ont été réalisées la première avec le nouveau terme source et une autre avec le terme de 
BATTJES \& JANSSEN (1978), formulation largement utilisée dans les modèles spectraux pour représenter la dissipation associée au déferlement bathymétrique. Les deux paramétrages sont globalement en bon accord avec les observations, le nouveau terme donnant des résultats légèrement meilleurs.

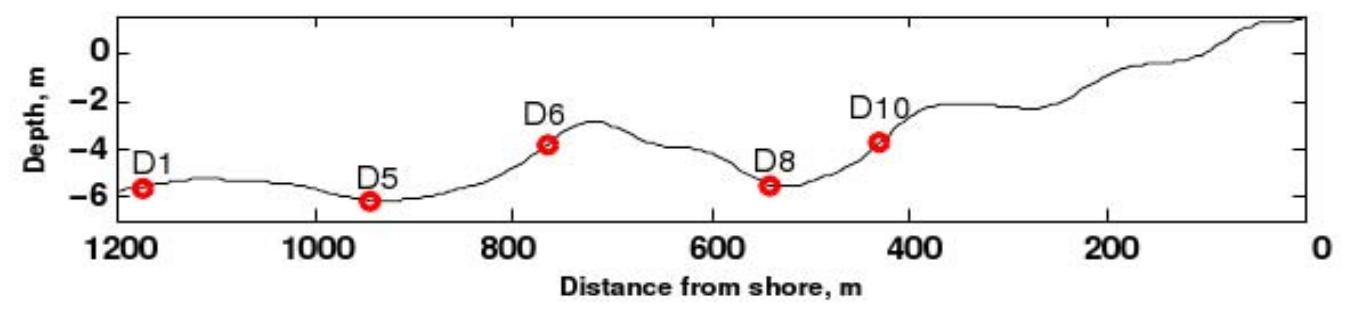

Figure 2. Profil de la bathymétrie utilisée pour la validation en eau peu profonde (campagne Duck94).

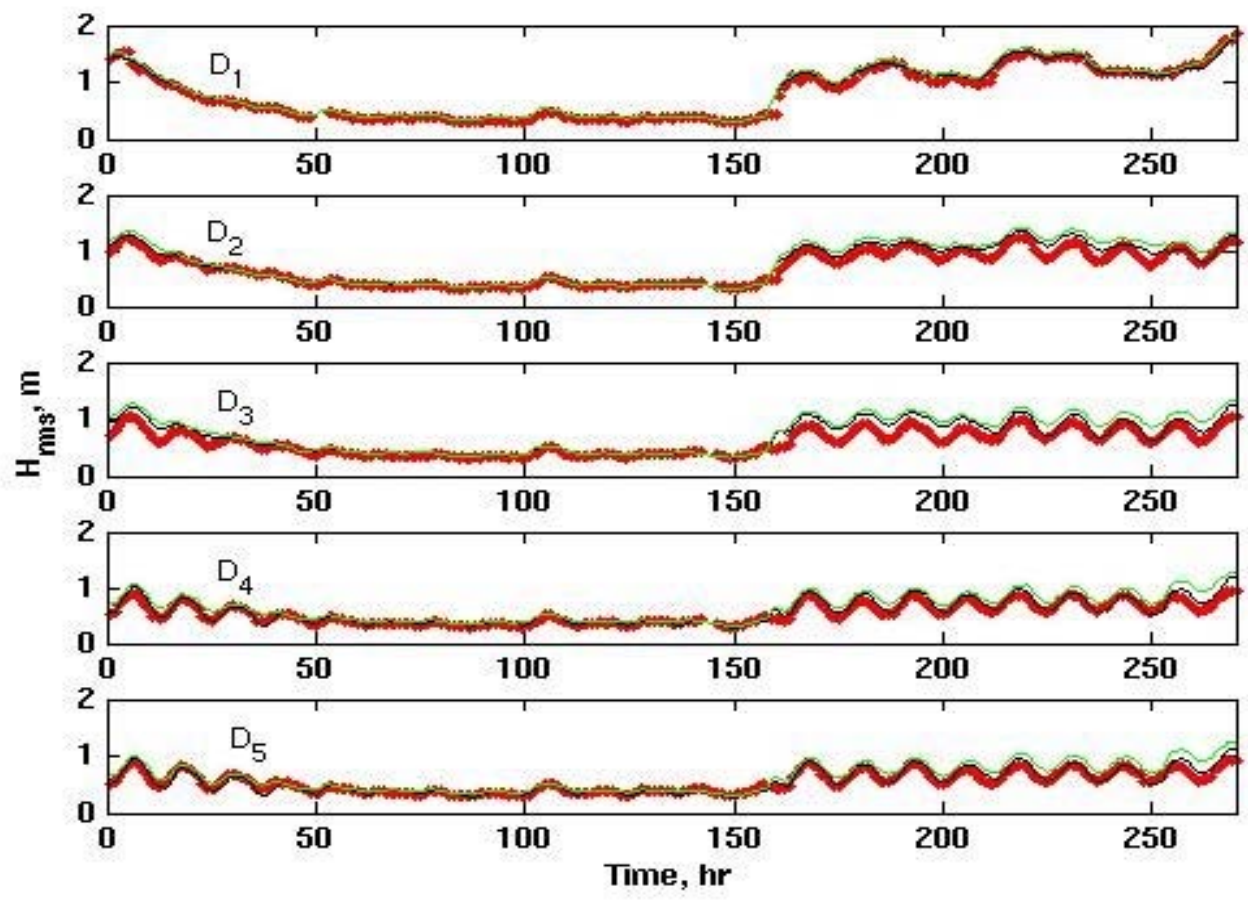

Figure 3. Hauteurs rms des vagues en fonction du temps, mesurées en différents points du profil. Points rouges : observations, ligne noire : nouveau paramétrage, ligne bleue : paramétrage de BATTJES \& JANSSEN (1978).

\section{Conclusions}

Nous avons présenté ici un nouveau terme source pour la dissipation d'énergie liée au déferlement. Ce terme est basé sur des paramétrages de grandeurs physiques observables et valides du large à la côte, la dissipation d'abord calculée dans le domaine 
physique et ensuite redistribuée sur les composantes spectrales. Les premières validations de ce terme, à la fois à l'échelle globale et en domaine côtier amènent des résultats encourageants. Certains points demandent cependant encore à être approfondis, comme par exemple le paramétrage de la densité de longueur de crête où encore les aspects directionnels qui n'ont pas encore été pris en compte.

\section{Remerciements}

Nous remercions Steve Elgar et Gerben Ruessink pour nous avoir fourni les observations de la campagne Duck94. Ces mesures été financées par l'US Office of Naval Research. J.F.F. remercie la DGA et le CNRS pour la bourse de recherche qui lui a été accordée. Ce travail est soutenu par un contrat ONR dans le cadre du U.S. National Ocean Partnership Program, et le Conseil Européen de la Recherche dans le cadre de la bourse jeune chercheur de F.A. pour le projet IOWAGA.

\section{Références bibliographiques}

ARDHUIN F., MARIE M., RASCLE N., FORGET P., ROLAND A. (2009). Observation and estimation of Lagrangian, Stokes and Eulerian currents induced by wind and waves at the sea surface, J. Phys. Oceanogr., vol. 39, no. 11, pp 2820-2838. doi:10.1175/2009JPO4169.1

ARDHUIN F., ROGERS E., BABANIN A.V., FILIPOT J.F., MAGNE R., ROLAND A., VAN DER WESTHUYSEN A., QUEFFEULOU P., LEFEVRE J.M, AOUF L., COLLARD F. (sous presse). Semi-empirical dissipation source function for wind-wave models: part I, definition, calibration and validation at global scales. J. Phys. Oceanogr., accepté.

BANNER M.L., BABANIN A.V., YOUNG I.R. (2000). Breaking probability for dominant waves on the sea surface. J. Phys. Oceanogr., vol. 30, pp 3145-3160. doi:10.1175/1520-0485(2000)030<3145:BPFDWO >2.0.CO;2

BANNER M.L., PEIRSON W.L. (2007). Wave breaking onset and strength for twodimensional deep-water wave groups. J. Fluid Mech. Vol. 585, pp 93-115. doi:10.1017/S0022112007006568

BATTJES J.A., JANSSEN J.P.F.M. (1978). Energy loss and set-up due to breaking of random waves. $16^{\text {th }}$ Coastal Eng. Conf., Hamburg, pp 569-587.

CHAWLA A., KIRBY J.T. (2002). Monochromatic and random wave breaking at blocking points. J. Geophys. Res., vol. 107(C7). doi:10.1029/2001JC001042

DUNCAN J.H. (1981). An experimental investigation of breaking waves produced by a towed hydrofoil. Proc. Roy. Soc. Lond. A, vol. 377, pp 331-348. doi:10.1098/rspa.1981.0127 ELGAR S., GUZA R.T., RAUBENHEIMER B., HERBERS T.H.C., GALLAGHER L. (1997). Spectral evolution od shoaling and breaking waves on a barred beach. J. Geophys. Res., vol. 102(C7), pp 15,797-15,805. doi:10.1029/97JC01010 
Thème 1 - Hydrodynamique côtière

FILIPOT J.F., ARDHUIN F., BABANIN F. (sous presse). A unified deep-to-shallow water wave-breaking probability parameterization. J. Geophys. Res.

HWANG L.S., DIVODKY D. (1970). Tsunami generation. J. Geophys. Res., vol. 75, pp 6802-6817. doi:10.1029/JC075i033p06802

MICHE A. (1944). Mouvements ondulatoires de la mer en profondeur croissante ou décroissante. forme limite de la houle lors de son déferlement. Application aux digues maritimes. Troisième partie. Forme et propriétés des houles limites lors du déferlement. Croissance des vitesses vers la rive. Annales des Ponts et Chaussées, Tome 114: pp 369-406.

MELVILLE W.K. (1994). Energy dissipation by breaking waves. J. Phys. Oceanogr., vol 24, pp 2041-2049. doi:10.1175/1520-0485(1994)024<2041:EDBBW>2.0.CO;2

PHILLIPS O.M., POSNER L.M., HANSEN J.P. (2001). High range resolution radar measurements of the speed distribution of breaking events in wind-generated ocean waves: surface impulse and wave energy dissipation rates. J. Phys. Oceanogr., vol. 31, pp 450-460. doi:10.1175/1520-0485(2001)031<0450:HRRRMO>2.0.CO;2

SCOTT N.S., WALSH E.J., HWANG P.A. (2005). Observations of Steep Wave Statistics in Open Ocean Waters. J. Atmos. Ocean Technol., vol. 22, pp 258-271. doi:10.1175/JTECH1702.1

THORNTON E.B., GUZA R.T. (1983). Transformation of wave height distribution. J. Geophys. Res., vol. 88, pp 5925-5938. doi:10.1029/JC088iC10p05925 